OPEN ACCESS

Edited by:

Rui Li,

University of Pennsylvania,

United States

Reviewed by:

Jingbo Pang,

University of Illinois at Chicago,

United States

Sandra Helena Poliselli Farsky,

University of São Paulo, Brazil

*Correspondence:

Ilhem Messaoudi

imessaou@uci.edu

Specialty section:

This article was submitted to

Inflammation,

a section of the journal

Frontiers in Immunology

Received: 11 June 2021 Accepted: 04 August 2021 Published: 20 August 2021

Citation:

Lewis SA, Sureshchandra S, Doratt B, Jimenez VA, Stull C, Grant KA and Messaoudi I (2021) Transcriptional, Epigenetic, and Functional Reprogramming of Monocytes From Non-Human Primates Following

Chronic Alcohol Drinking.

Front. Immunol. 12:724015. doi: 10.3389/fimmu.2021.724015

\section{Transcriptional, Epigenetic, and Functional Reprogramming of Monocytes From Non-Human Primates Following Chronic Alcohol Drinking}

\author{
Sloan A. Lewis ${ }^{1,2}$, Suhas Sureshchandra ${ }^{1,2}$, Brianna Doratt ${ }^{1,2}$, Vanessa A. Jimenez ${ }^{3}$, \\ Cara Stull ${ }^{3}$, Kathleen A. Grant ${ }^{3}$ and llhem Messaoudi ${ }^{1,2 *}$

\begin{abstract}
${ }^{1}$ Department of Molecular Biology and Biochemistry, University of California, Irvine, CA, United States, ${ }^{2}$ Institute for Immunology, University of California, Irvine, CA, United States, ${ }^{3}$ Oregon National Primate Research Center, Oregon Health
\end{abstract} \\ and Science University, Beaverton, OR, United States
}

Chronic heavy drinking (CHD) of alcohol is a known risk factor for increased susceptibility to bacterial and viral infection as well as impaired wound healing. Evidence suggests that these defects are mediated by a dysregulated inflammatory response originating from myeloid cells, notably monocytes and macrophages, but the mechanisms remain poorly understood. Our ability to study CHD is impacted by the complexities of human drinking patterns and behavior as well as comorbidities and confounding risk factors for patients with alcohol use disorders. To overcome these challenges, we utilized a translational rhesus macaque model of voluntary ethanol self-administration that closely recapitulates human drinking patterns and chronicity. In this study, we examined the effects of CHD on blood monocytes in control and CHD female macaques after 12 months of daily ethanol consumption. While monocytes from CHD female macaques generated a hyperinflammatory response to ex vivo LPS stimulation, their response to $E$. coli was dampened. In depth scRNA-Seq analysis of purified monocytes revealed significant shifts in classical monocyte subsets with accumulation of cells expressing markers of hypoxia (HIF1A) and inflammation (NFkB signaling pathway) in CHD macaques. The increased presence of monocyte subsets skewed towards inflammatory phenotypes was complemented by epigenetic analysis, which revealed higher accessibility of promoter regions that regulate genes involved in cytokine signaling pathways. Collectively, data presented in this manuscript demonstrate that CHD shifts classical monocyte subset composition and primes the monocytes towards a more hyper-inflammatory response to LPS, but compromised pathogen response.

Keywords: alcohol, inflammation, monocytes, nonhuman primates, scRNA seq, ATAC-Seq 


\section{INTRODUCTION}

Alcohol consumption is widespread in the United States with $85 \%$ of individuals ages 18 and older engaging in this behavior. While most of these individuals are considered moderate drinkers, $7 \%$ are classified as heavy alcohol users (National Survey on Drug Use and Health, 2015). Chronic heavy alcohol consumption or chronic heavy drinking (CHD) is associated with multiple adverse health effects including increased incidence of cardiac disease $(1,2)$, certain types of cancer (36), liver cirrhosis (7), and sepsis (8), making it the third leading preventable cause of death in the United States (9). CHD is also associated with higher susceptibility to bacterial and viral infections including pneumonia and tuberculosis $(10,11)$, hepatitis C virus, and $\operatorname{HIV}(12,13)$. Moreover, CHD compromises tissue repair, resulting in reduced post-operative healing and poor trauma recovery outcomes $(14,15)$. These observations strongly suggest that $\mathrm{CHD}$ dysregulates immunity and host defense.

While CHD can modulate the function of many immune cell populations, data from several laboratories indicate that the most dramatic and consistent changes are evident in the innate immune branch, specifically in myeloid cells (monocytes, macrophages, dendritic cells, and neutrophils) (16-18). Monocytes are relatively short-lived phagocytic cells that circulate in the blood, are constantly repopulated from bone marrow progenitors, and can quickly respond to infection or inflammation by extravasation into tissue and differentiation into tissue-resident macrophages (19). A tightly regulated inflammatory response by monocytes is required for effective infection clearance and tissue repair (20).

Alcohol consumption has been shown to disrupt monocyte and macrophage responses in a dose and time dependent manner (16). Specifically, acute alcohol treatment of purified primary human monocytes, rodent monocyte-derived macrophages, or human monocytic cell lines results in decreased production of inflammatory mediators including TNF $\alpha$ following stimulation with lipopolysaccharide (LPS), a TLR4 agonist (21-23). In rodents, acute in vivo (24) exposure to ethanol increases production of the anti-inflammatory cytokine IL-10 through activation of STAT3. Similarly, in healthy human subjects, an acute binge of alcohol increased IL-10 and decreased IL-1 $\beta$ production (25). In contrast, prolonged exposure to alcohol results in increased production of pro-inflammatory mediators, notably TNF $\alpha$ in response to LPS or phorbol myristate acetate (PMA)/ionomycin stimulation (22, 26) potentially due to enhanced activation of NFKB and ERK kinases (27). In line with these observations, monocytes from patients with alcoholic liver disease (ALD) as well as tissue resident macrophage populations including Kupffer cells (28), microglia (29), alveolar macrophages (30), and splenic macrophages $(17,31)$ produce higher levels of TNF- $\alpha$ at resting state as well as in response to LPS (32). The enhanced inflammatory response by tissue-resident myeloid cells in the context of CHD is linked to organ damage, most notably in the liver (33) but also the intestine $(34)$, brain $(35,36)$, and lungs (37).
Despite the studies described above, our understanding of the mechanisms by which chronic alcohol consumption reprograms circulating monocytes and tissue-resident macrophages remains incomplete. Some studies have suggested that CHD-induced gut "leakiness" and translocation of bacterial products including LPS across the gut barrier into the circulation could lead to chronic activation and subsequently organ damage (34). Whether monocytes in circulation are activated prior to organ damage by circulating bacterial products, ethanol, ethanol metabolites, or a combination of these factors remains unanswered.

Data from clinical studies are confounded by self-reported alcohol intake, the use of nicotine, recreational or illicit drugs, nutritional deficiencies, and presence of organ damage (38). Addressing these questions requires access to a reliable animal model that recapitulates critical aspects of human CHD. Therefore, to better model human $\mathrm{CHD}$ and relate immune response of peripheral monocytes to quantified alcohol intakes in the absence of confounders listed above, we leveraged a rhesus macaque model of chronic voluntary ethanol self-administration $(39,40)$. Using this model, we reported that CHD disrupts the resting transcriptome and results in heightened inflammatory responses by peripheral blood mononuclear cells (PBMC) from male and female macaques $(18,41)$. Additionally, splenic macrophages from CHD monkeys generate a hyperinflammatory response following LPS stimulation that is accompanied by increased chromatin accessibility at promoters and intergenic regions that regulate genes important for inflammatory responses (17).

In this study, we examine how CHD alters the ability of blood monocytes to respond properly to stimulation. We show that $\mathrm{CHD}$ is associated with increased numbers of circulating monocytes that exhibit a heightened transcriptional and immune mediator response to LPS stimulation, but lower response to bacterial pathogens. To determine the molecular mechanisms of this dysregulated response, we profiled the transcriptome of the monocytes by single cell RNA-Seq and their epigenetic landscape by ATAC-Seq. Our results indicate that CHD significantly alters the epigenetic and transcriptional profiles of circulating monocytes, priming them towards an inflammatory state but altering their effector function to towards pathogens.

\section{METHODS AND MATERIALS}

\section{Animal Studies and Sample Collection}

These studies used samples from a non-human primate model of voluntary ethanol self-administration established through schedule-induced polydipsia $(39,40,42)$. Briefly, in this model, rhesus macaques are introduced to a $4 \% \mathrm{w} / \mathrm{v}$ ethanol solution during a 90 -day induction period followed by concurrent access to the $4 \% \mathrm{w} / \mathrm{v}$ solution and water for 22 hours/day for one year. During this time, the macaques adopt a stable drinking phenotype defined by the amount of ethanol consumed per day and the pattern of ethanol consumption (g/kg/day) (39). Blood samples were taken from the saphenous vein every 5-7 days at $7 \mathrm{hrs}$ after the onset of the $22 \mathrm{hr} /$ day access to ethanol and 
assayed by headspace gas chromatography for blood ethanol concentrations (BECs).

For these studies, blood samples were collected from 9 female and 3 male rhesus macaques (average age $5.68 \mathrm{yrs}$ ), with 5 animals serving as controls and 8 classified as chronic heavy drinkers (CHD) based on 12 months of ethanol selfadministration (tissue and drinking data obtained from the Monkey Alcohol Tissue Research Resource: www.matrr.com). These cohorts of animals (Cohorts 6 and $7 \mathrm{a}$ on matr.com) were described in two previous studies of innate immune system response to alcohol $(18,41)$. Peripheral Blood Mononuclear Cells (PBMC) were isolated by centrifugation over histopaque (Sigma, St Louis, MO) as per manufacturer's protocol and cryopreserved until they could be analyzed as a batch. The average daily ethanol intake for each animal is outlined in Supplementary Table 1. CHD animals did not show signs of overt liver damage as measured by alanine transaminase (ALT) and aspartate transaminase (AST) enzyme levels [Supplementary Figure 2G and (43)].

\section{LAL and IgM Assays}

Endotoxin-core antibodies in plasma samples were measured using an enzyme-immunoassay technique (ELISA) after 12 months of alcohol consumption using EndoCab IgM ELISA kit (Hycult Biotech, Catalog\# HK504-IgM). Plasma samples were diluted 50x.

Circulating endotoxin was measured from plasma using a Limulus amebocyte lysate (LAL) assay (Hycult Biotech) following the manufacturers protocol.

\section{Flow Cytometry Analysis}

$1-2 \times 10^{6}$ PBMC were stained with the following surface antibodies (2 panels) against: CD3 (BD Biosciences,SP34, FITC), CD20 (Biolegend, 2H7, FITC), HLA-DR (Biolegend, L243, APC-Cy7), CD14 (Biolegend, M5E2, AF700), CD16 (Biolegend, 3G8, PB), TLR4 (Biolegend, HTA125, APC), TLR2 (Biolegend, TLR2.1, A488), CD40 (Biolegend, 5C3, BV510), CD163 (Biolegend, GHI/61, PerCp-Cy5.5), CD86 (Biolegend, IT2.2, BV605), CD80 (Biolegend, 2D10, PE), CX3CR1 (Biolegend, 2A9-1, PE), CCR7 (Biolegend, GO43H7, BV510), and CCR5 (Biolegend, J418F1, PE-Cy7) and live/dead Sytox Aadvanced (Invitrogen). Monocytes were defined as CD3CD20-HLA-DR+CD14+. All samples were acquired with an Attune NxT Flow Cytometer (ThermoFisher Scientific, Waltham, MA) and analyzed using FlowJo software (Ashland, OR). Median Fluorescence Intensities (MFI) for all markers within the CD14+ monocyte gate were tested for significant differences using an unpaired t-test with Welch's correction on Prism 7 (GraphPad, San Diego, CA).

\section{CD14 MACS Bead Isolation and Purity}

CD14+ monocytes were purified from freshly thawed PBMC using CD14 antibodies conjugated to magnetic microbeads per manufacturers recommendations (Milyenyi Biotec, San Diego, CA). Efficiency of the positive selection of monocytes was assessed by flow cytometry where purity (CD14+HLA-DR+) averaged $87 \%(\mathrm{SEM} \pm 1.6)$.

\section{Monocyte Stimulation Assays}

$1 \times 10^{6}$ freshly thawed PBMC or $1 \times 10^{5}$ purified CD $14+$ monocytes were cultured in RPMI supplemented with $10 \%$ FBS with or without $100 \mathrm{ng} / \mathrm{mL}$ LPS (TLR4 ligand, E. coli 055:B5; Invivogen, San Diego CA) for 16 or 6 hours, respectively, in 96-well tissue culture plates at $37 \mathrm{C}$ in a $5 \% \mathrm{CO}_{2}$ environment. $3.5 \times 10^{4}$ purified CD14+ monocytes were cultured in RPMI supplemented with $10 \%$ FBS with or without $6 \times 10^{5} \mathrm{cfu} / \mathrm{mL}$ heat-killed $E$. coli (Escherichia coli (Migula) Castellani and Chalmers ATCC 11775 ) for 16 hours in 96-well tissue culture plates at 37C in a $5 \% \mathrm{CO}_{2}$ environment. Plates were spun down: supernatants were used to measure production of immune mediators and cell pellets were resuspended in Qiazol (Qiagen, Valencia CA) for RNA extraction. Both cells and supernatants were stored at $-80 \mathrm{C}$ until they could be processed as a batch.

\section{Luminex Assay}

Immune mediators in the supernatants from PBMC or purified monocytes stimulated with LPS were measured using a 30-plex panel measuring levels of cytokines (IFN $\gamma$, IL-1b, IL-2, IL4, IL-6, IL-7, IL-12, IL-15, IL-17, IL-18, IL-23, TNFo, IL-1RA, IFN-b, and IL-10), chemokines (MCP-1, MIP-1a, MIP-1b, Eotaxin, IL8, MIG, I-TAC, BCA-1, IP-10, and SDF-1a), and other factors (PD-L1, PDGF-BB, S100B, GM-CSF, and VEGF-A) validated for NHP (R\&D Systems, Minneapolis, MN, USA). Standard curves were generated using 5-parameter logistic regression using the $\mathrm{xPONENT}^{\mathrm{TM}}$ software provided with the MAGPIX instrument (Luminex, Austin TX).

Immune mediators in the supernatants from monocytes stimulated with $E$. coli were measured using a more sensitive ProcartaPlex 31-plex panel measuring levels of cytokines (IFN $\alpha$, IFN $\gamma$, IL-1 $\beta$, IL-10, IL-12p70, IL-15, IL-17A, IL-1RA, IL-2, IL-4, IL-5, IL-6, IL-7, MIF, and TNF $\alpha$ ), chemokines (BLC(CXCL13), Eotaxin (CCL11), I-TAC(CXCL11), IL-8(CXCL8), IP-10 (CXCL10), MCP-1(CCL2), MIG(CXCL9), MIP-1a(CCL3), MIP-1b(CCL4)), growth factors (BDNF, G-CSF, GM-CSF, PDGF-BB, VEGF-A) and other factors (CD40L, Granzyme B) (Invitrogen, Carlsbad, CA). Differences in induction of proteins post stimulation were tested using unpaired t-tests with Welch's correction. Dose-dependent responses were modeled based on $\mathrm{g} / \mathrm{kg} /$ day ethanol consumed and tested for linear fit using regression analysis in Prism (GraphPad, San Diego CA). Raw data included in Supplementary Table 2.

\section{RNA Isolation and Library Preparation}

Total RNA was isolated from PBMC or purified CD14+ monocytes from female macaques using the mRNeasy kit (Qiagen, Valencia CA) following manufacturer instructions and quality assessed using Agilent 2100 Bioanalyzer. Libraries from PBMC RNA were generated using the TruSeq Stranded RNA LT kit (Illumina, San Diego, CA, USA). Libraries from purified CD14+ monocytes RNA were generated using the NEBnext Ultra II Directional RNA Library Prep Kit for Illumina (NEB, Ipswitch, MA, USA), which allows for lower input concentrations of RNA (10ng). For both library prep kits, rRNA depleted RNA was fragmented, converted to doublestranded cDNA and ligated to adapters. The roughly $300 \mathrm{bp}-$ 
long fragments were then amplified by PCR and selected by size exclusion. Libraries were multiplexed and following quality control for size, quality, and concentrations, were sequenced to an average depth of 20 million $100 \mathrm{bp}$ reads on the HiSeq 4000 platform.

\section{Bulk RNA-Seq Data Analysis}

RNA-Seq reads were quality checked using FastQC (https:// www.bioinformatics.babraham.ac.uk/projects/fastqc/), adapter and quality trimmed using TrimGalore(https://www. bioinformatics.babraham.ac.uk/projects/trim_galore/), retaining reads at least $35 \mathrm{bp}$ long. Reads were aligned to Macaca mulatta genome (Mmul_8.0.1) based on annotations available on ENSEMBL (Mmul_8.0.1.92) using TopHat (44) internally running Bowtie2 (45). Aligned reads were counted gene-wise using GenomicRanges (46), counting reads in a strand-specific manner. Genes with low read counts (average <5) and nonprotein coding genes were filtered out before differential gene expression analyses. Read counts were normalized using RPKM method for generation of PCA and heatmaps. Raw counts were used to test for differentially expressed genes (DEG) using edgeR (47), defining DEG as ones with at least two-fold up or down regulation and an FDR controlled at 5\%. Functional enrichment of gene expression changes in resting and LPS-stimulated cells was performed using Metascape (48) and DAVID (49). Networks of functional enrichment terms were generated using Metascape and visualized in Cytoscape (50). Transcription factors that regulate expression of DEG were predicted using the ChEA3 (51) tool using ENSEML ChIP database.

\section{X scRNA-Seq Data Analysis}

PBMC from control $(n=3)$ and $\mathrm{CHD}(n=3)$ female macaques were thawed and stained with anti-CD14, HLA-DR antibodies and sorted for live CD14+/HLA-DR+ cells on a BD FACSAria Fusion on the same day. Sorted monocytes were pooled and resuspended at a concentration of 1200 cells/ul and loaded into the 10X Chromium gem aiming for an estimated 10,000 cells per sample. cDNA amplification and library preparation (10X v3 chemistry) were performed according to manufacturer protocol and sequenced on a NovaSeq S4 (Illumina) to a depth of $>50,000$ reads/cell.

Sequencing reads were aligned to the Mmul_8.0.1 reference genome using cellranger v3.1 (52) (10X Genomics). Quality control steps were performed prior to downstream analysis with Seurat, filtering out cells with fewer than 200 unique features and cells with greater than $20 \%$ mitochondrial content. Control and CHD datasets were down sampled to 4680 cells each and integrated in Seurat (53) using the IntegrateData function. Data normalization and variance stabilization were performed, correcting for differential effects of mitochondrial and cell cycle gene expression levels. Clustering was performed using the first 20 principal components. Small clusters with an over-representation of $\mathrm{B}$ and $\mathrm{T}$ cell gene expression were removed for downstream analysis. Clusters were visualized using uniform manifold approximation and projection (UMAP) and further characterized into distinct monocyte subsets using the FindMarkers function (Supplementary Table 3).

\section{Pseudo-Temporal Analysis}

Pseudotime trajectory monocytes was reconstructed using Slingshot (54). The UMAP dimensional reduction performed in Seurat was used as the input for Slingshot. For calculation of the lineages and pseudotime, the most abundant classical monocyte cluster, MS1, was set as the root state.

\section{Differential Expression Analyses}

Differential expression analysis (CHD to Control) was performed using DESeq under default settings in Seurat. Only statistically significant genes (Fold change cutoff $\geq 1.2$; adjusted $\mathrm{p}$-value $\leq 0.05)$ were included in downstream analysis.

\section{Module Scoring and Functional Enrichment}

For gene scoring analysis, we compared gene signatures and pathways from KEGG (https://www.genome.jp/kegg/pathway. html) (Supplementary Table 4) in the monocytes using Seurat's AddModuleScore function. Over representative gene ontologies were identified using 1-way, 2-way or 4-way enrichment of differential signatures using Metascape (48). All plots were generated using ggplot2 and Seurat.

\section{ATAC-Seq Library Preparation}

Following the Omni-ATAC protocol, $2 \times 10^{4}$ purified monocytes from female macaques were lysed in lysis buffer $(10 \mathrm{mM}$ Tris- $\mathrm{HCl}$ ( $\mathrm{pH} 7.4$ ), $10 \mathrm{mM} \mathrm{NaCl}, 3 \mathrm{mM} \mathrm{MgCl}, 10 \% \mathrm{~Np}-40,10 \%$ Tween, and $1 \%$ Digitonin) on ice for 3 minutes (55). Immediately after lysis, nuclei were spun at $500 \mathrm{~g}$ for 10 minutes at $4 \mathrm{C}$ to remove supernatant. Nuclei were then incubated with Tn5 transposase for 30 minutes at 37C. Tagmented DNA was purified using AMPure XP beads (Beckman Coulter, Brea, CA) and PCR was performed to amplify the library under the following conditions: $72 \mathrm{C}$ for $5 \mathrm{~min}$; 98 for $30 \mathrm{~s}$; 5 cycles of $98 \mathrm{C}$ for $10 \mathrm{~s}, 63 \mathrm{C}$ for $30 \mathrm{~s}$, and $72 \mathrm{C}$ for $1 \mathrm{~min}$; hold at $4 \mathrm{C}$. Libraries were then purified with warm AMPure XP beads and quantified on a Bioanalyzer (Agilent Technologies, Santa Clara CA). Libraries were multiplexed and sequenced to a depth of 50 million $100 \mathrm{bp}$ paired reads on a NextSeq (Illumina).

\section{ATAC-Seq Data Analysis}

Paired ended reads from sequencing were quality checked using FastQC and trimmed to a quality threshold of 20 and minimum read length 50. Trimmed reads were aligned to the Macaca Mulatta genome (Mmul_8.0.1) using Bowtie2 (-X 2000 -k 1 very-sensitive -no-discordant -no-mixed). Reads aligning to mitochondrial genome were removed using Samtools and PCR duplicate artifacts were removed using Picard. Samples from each group were concatenated to achieve greater than $5 \times 10^{6}$ nonduplicate, non-mitochondrial reads per group.

Accessible chromatin peaks were called using Homer's findPeaks function $(56)($ FDR $<0.05)$ and differential peak analysis was performed using Homer's getDifferentialPeaks function $(\mathrm{P}<0.05)$. Genomic annotation of open chromatin regions in monocytes and differentially accessible regions (DAR) with CHD was assigned using ChIPSeeker (57). Promoters were defined as $-1000 \mathrm{bp}$ to $+100 \mathrm{bp}$ around the transcriptional start 
site (TSS). Functional enrichment of open promoter regions was performed using Metascape (http://metascape.org).

Due to the lack of available macaque annotation databases, distal intergenic regions from the macaque assembly were converted to the human genome (hg19) coordinates using the UCSC liftOver tool. Cis-Regulatory roles of these putative enhancer regions were identified using GREAT (http://great. stanford.edu/public/html/). Transcription factor motif analysis was performed using Homer's findMotifs function with default parameters. Promoter regions for every annotated macaque gene were defined in ChIPSeeker as -1000 bp to +100 bp relative to the TSS. A counts matrix was generated for these regions using featureCounts (58), where pooled bam files for each group were normalized to total numbers of mapped reads.

\section{Statistical Analysis}

All statistical analyses were conducted in Prism 7(GraphPad). Data sets were first tested for normality. Two group comparisons were carried out an unpaired t-test with Welch's correction. Differences between 4 groups were tested using one-way ANOVA $(\alpha=0.05)$ followed by Holm Sidak's multiple comparisons tests. Error bars for all graphs are defined as \pm SEM. Linear regression analysis compared significant shifts in curve over horizontal line, with spearman correlation coefficient reported. Statistical significance of functional enrichment was defined using hypergeometric tests. P-values less than or equal to 0.05 were considered statistically significant. Values between 0.05 and 0.1 are reported as trending patterns.

\section{RESULTS}

\section{Chronic Heavy Drinking Results in Enhanced Responses to LPS by PBMC}

Recent bulk RNA seq analysis of resting PBMC obtained from female rhesus macaques after 12 months of chronic heavy drinking (CHD) indicated that most of the differential gene expression originated from innate immune cells [monocytes and dendritic cells (DCs)] (18). Moreover, studies using PBMC from male macaques showed increased responses to ex vivo LPS stimulation after 12 months of chronic heavy ethanol consumption (41). To assess if CHD also led to changes in inflammatory responses by circulating innate immune cells in female macaques, PBMC obtained from CHD $(n=6)$ and control $(n=3)$ females were stimulated ex vivo with LPS for 16 hours followed by measurement of immune mediator production by Luminex assay and transcriptional changes by bulk RNA Seq (Supplementary Figure 1A). LPS stimulation resulted in a robust inflammatory response (TNF $\alpha$, IL-6, IL-18, IL-4, IL-8, GMCSF and S100B) by PBMC from both CHD and control animals (Figure 1A). However, PBMC from CHD animals produced significantly elevated levels of additional inflammatory mediators notably IL-1 $\beta$, IL-12, IFN $\gamma$, CCL3, and CCL4 compared to their unstimulated condition (Figure 1A). Moreover, production of inflammatory cytokines IL-6, IL-23 and to lesser extent soluble PD-L1 was higher by CHD PBMC following LPS stimulation compared to controls (Figure 1A). In addition, levels of TNF $\alpha$, CCL4, IL-6, IL-15, IL-23, and sPDL1 were positively correlated with ethanol dose (Figure 1B and Supplementary Figure 1B).

We next assessed transcriptional differences in response to LPS between the two groups. Differential analysis indicated a heightened response to LPS by CHD PBMC relative to control PBMC (Figure 1C). While signatures of immune activation were observed in both groups following LPS stimulation, DEGs upregulated in PBMC from controls enriched more significantly to pathways involved in antiviral immunity ("response to IFN $\gamma$ " and "response to virus"), whereas DEGs upregulated in PBMC from CHD animals enriched more significantly to leukocyte activation and inflammation pathways (Figure 1D and Supplementary Figure 1C). Enrichment of the downregulated genes shows significant enrichment to GO terms associated with chemotaxis, leukocyte differentiation and metabolism in the PBMC from control animals (Figure 1E and Supplementary Figure 1D). These findings indicate disruption of innate immune responses with $\mathrm{CHD}$ and further highlight that the heightened inflammatory response to LPS by PBMC is not restricted to male macaques.

\section{Chronic Heavy Drinking Impacts Monocyte Frequencies and Functional Responses to LPS Stimulation}

To measure changes in frequencies and phenotypes of monocyte subsets with CHD in female macaques, we profiled PBMC by flow cytometry. Interestingly, while the total white blood cell numbers were unchanged with CHD (18), the proportions of monocytes within PBMC from CHD macaques were significantly elevated (Figure 2A). However, the relative distribution of the monocyte sub-populations were comparable between the groups (Supplementary Figures 2A, B). We profiled several TLRs, chemokine, and activation receptors on monocytes by flow cytometry, but found no significant differences (Supplementary Figure 2C). To assess whether the heightened inflammatory response detected in PBMC was due to increased numbers of monocytes or cell-intrinsic changes caused by $\mathrm{CHD}$, monocytes were purified from each group and subjected to bulk RNA-Seq at resting state and after LPS stimulation (Supplementary Figure 1A). A modest number of DEG were detected at resting state (58 upregulated and 112 downregulated) between $\mathrm{CHD}$ and control monocytes (Figure 2B). The DEG upregulated with $\mathrm{CHD}$ enriched to pathways associated with inflammatory response such as "pattern recognition receptor activity" (e.g. NLRP3) and "cytokine production" (e.g. FCN1) (Figure 2C, and Supplementary Figure 2D). DEG downregulated with CHD mapped to gene ontology (GO) terms "activation of immune response" (e.g. IFNG) "lymphocyte proliferation" (e.g. HSPD1) and "chemotaxis" (e.g. CCR7) (Figure 2C). One potential mechanism for this enhanced monocyte transcriptional activation at resting state could be increased microbial products in circulation due to altered barriers (31). Therefore, we measured circulating levels of bacterial endotoxin (LAL) and 


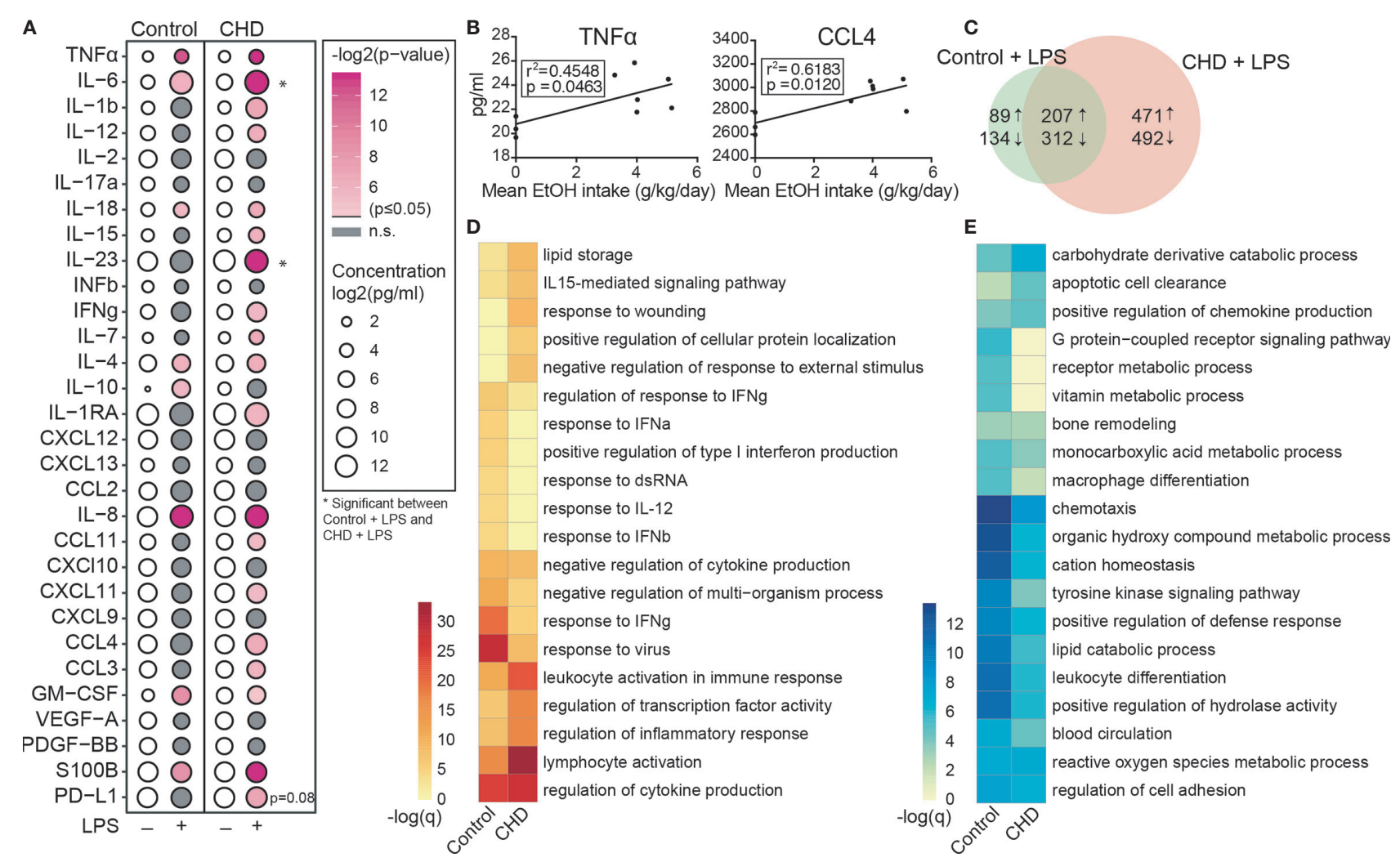

FIGURE 1 | CHD induced enhanced innate immune response in the periphery. PBMC from control $(n=3)$ and chronic heavy drinking $(C H D)(n=6)$ female macaques were stimulated with LPS for 16 hours. (A) Bubble plot representing immune factor production (pg/ml) in the presence or absence of LPS stimulation of PBMC from control and CHD animals. The size of each circle represents the $\log _{2}$ mean concentration of the indicated secreted factor and the color denotes the -log 2 transformed $p$ value with the darkest pink representing the most significant value. The $p$-values were calculated between the unstimulated and stimulated conditions for each group by One-way ANOVA and a p-value cut-off of 0.05 was set. White circles indicate non-significant p-value. * indicates significance between control and $\mathrm{CHD}$ for each stimulation condition. (B) Scatter plots showing Spearman correlation between average EtOH dose (grams EtOH/kg body weight/day) and concentration $(\mathrm{pg} / \mathrm{ml})$ of the secreted factors TNF $\alpha$ and CCL4. (C) Venn diagram comparing LPS-induced DEG in controls and CHD PBMC. (D, E) Heatmaps of significant GO terms to which upregulated (D) and downregulated (E) DEG identified following LPS stimulation of PBMC from controls and CHD animals enriched using Metascape. The scales of the heatmaps are -log (q-values) associated with the enrichment to selected pathways.

IgM bound endotoxin. We found slightly increased levels of circulating LAL with CHD, but no changes in IgM-bound endotoxin (Supplementary Figures 2E, F). Additionally, levels of alanine transaminase (ALT) and aspartate transaminase (AST) levels, both of which are indicators of liver damage, were also comparable between the two groups (Supplementary Figure 2G).

Secreted levels of cytokines, chemokines, and growth factors were measured after 6-hour incubation in the presence or absence of LPS by Luminex assay. No significant differences in immune mediators' secretion were noted in the non-stimulated conditions. As observed for PBMC, significantly enhanced production of pro-inflammatory cytokines TNF $\alpha$, IL-6, and IL15, chemokines CCL4, and CXCL11, and to a lesser extent IL-4 and IL-7 by CHD monocytes were noted (Figure 2D and Supplementary Figure 3A). The LPS response was also assessed at the transcriptional level using RNA-Seq. Principal component analysis (PCA) showed that stimulation accounts for the bulk of the transcriptional changes (PC1, 75\% of the differences) (Supplementary Figure 3B) with 357 and 262 DEG between CHD and controls in the NS, and LPS conditions, respectively (Supplementary Figures 3C, D). The DEG from these comparisons showed that genes associated with myeloid inflammatory pathways were upregulated (TNFSF21, MMP2, TLR2, MMP1) while genes associated with adaptive immune activation were downregulated (IL21R, CD40, $M A M U-D O A, C C R 6)$ in CHD compared to control monocytes (Supplementary Figures 3C, D).

We then identified DEG in the LPS relative to NS condition for each group. A greater number of DEG was detected in the CHD group, with a large overlap between the two groups, and few DEG detected solely in the control group (Figure 2E). As expected, DEG common between the two groups enriched to GO terms associated with monocyte activation including "regulation cytokine production", "leukocyte migration", and "JAK-STAT cascade" (Figure 2F). The DEG detected only in control animals mapped to "Carbohydrate catabolic process". The DEG unique to monocytes from CHD animals mapped to cytokine 
A

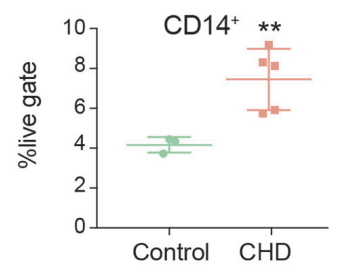

B

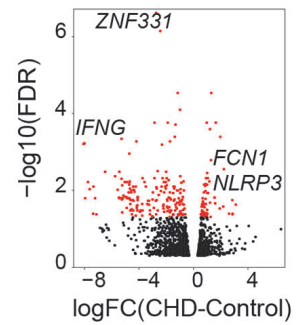

C

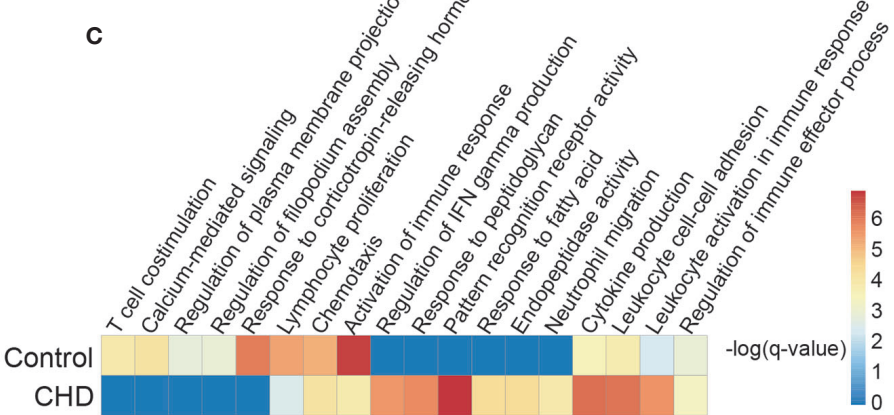

E
D

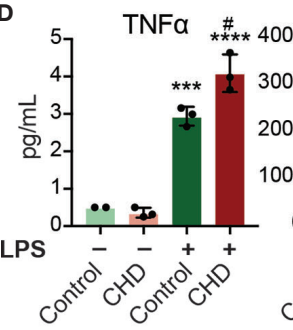

Upregulated with LPS both

$\mathrm{CHD}$
IL-6 \# ${ }_{* \star *}^{*} 5_{7} \quad$ IL-15
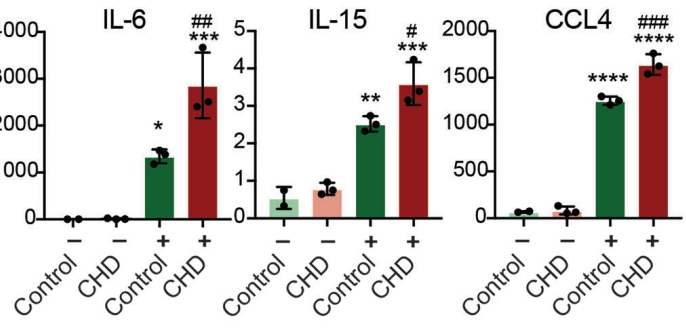

\section{G}

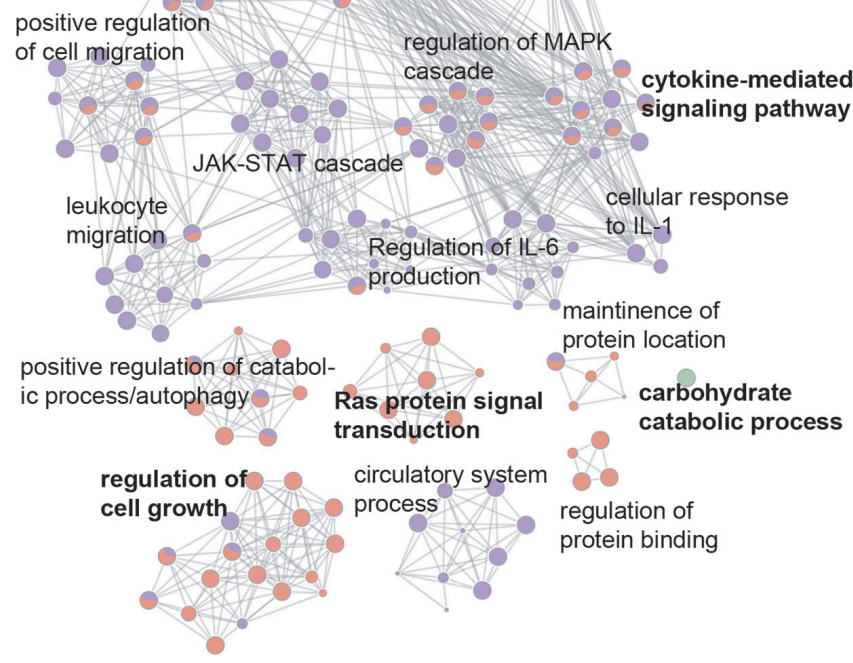
cellular TNF

cell-adhesion regulation of cytokine

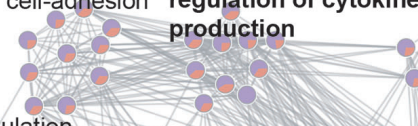
regulation of MAPK

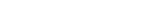

DEGs with LPS stimulation: Control $\mathrm{CHD}$

$47 \uparrow 514 \uparrow \quad 750 \uparrow$

FIGURE 2 | Impact of CHD on the monocyte functional and transcriptomic response to LPS. (A) Abundance of live CD14+ cells in PBMC from control $(n=3)$ and $\mathrm{CHD}(\mathrm{n}=5)$ female macaques measured by flow cytometry. Significance calculated by t-test with Welch's correction. (B) Volcano plot representing up- and downregulated differentially expressed genes (DEG) with CHD in resting monocytes. Red = significant with an FDR $\leq 0.05$ and fold change $\geq 2$. (C) Heatmap of significant GO terms (identified using Metascape) to which DEGs downregulated and upregulated with CHD in monocytes enriched. The scales of the heatmaps are - $\log (q$-values) associated with the enrichment to selected pathways. (D) Purified monocytes were stimulated with LPS for 6 hours and supernatants were analyzed by Luminex assay. Bar plots showing concentration (pg/ml) of selected immune mediators (TNF $\alpha$, IL-6, IL-15, CCL4) $\left({ }^{*} \mathrm{p}<0.05,{ }^{* \star} \mathrm{p}<0.01,{ }^{* \star *} \mathrm{p}<0.001,{ }^{\star \star \star *} \mathrm{p}<\right.$ 0.0001 between LPS and unstimulated condition, ${ }^{\#} p<0.05$, ${ }^{\# \#} p<0.01$, ${ }^{\# \# \#} p<0.001$ between CHD and control for each stimulation condition calculated by Oneway ANOVA). (E) Venn diagram showing overlap between DEG identified in control and CHD monocytes after LPS stimulation. (F) Network visualization of functional enrichment analysis of DEG upregulated with LPS in monocytes from controls only, CHD only, and both using Metascape. The size of each node represents the number of DEGs associated with a given gene ontology (GO) term and the pie chart filling represents relative proportion of DEGs from each group that enriched to that GO term. Similar GO terms are clustered together and are titled with the name of the most statistically significant GO term within that cluster. The gray lines denote shared interactions between GO terms. Density and number of gray lines indicates the strength of connections between closely related GO terms. (G) Heatmap of average normalized expression of genes associated with inflammatory response pathways. 
production and signaling pathways (Figure 2F). The expression of inflammatory genes was broadly upregulated in the $\mathrm{CHD}$ monocytes following LPS stimulation including CEBPB, CCL20, CCL4, STAT3, FOS, S100A8, HIF1A, and TLR4 (Figure 2G). Additional predictive analysis revealed that LPS-responsive DEGs detected in the CHD monocytes are regulated by canonical transcription factors NFKB2, RELB, and JUNB with over 250 genes mapping to each (Supplementary Figure 3E).

\section{Chronic Heavy Drinking Alters the Monocyte Transcriptome and Cell Subset Distribution at the Single Cell Level}

To gain a deeper understanding of the heightened activation state of monocytes with $\mathrm{CHD}$, we performed single cell RNA sequencing (scRNA-Seq) on sorted CD14+HLA-DR+ monocytes from $\mathrm{CHD}$ and control female macaques $(n=3$ pooled samples/group). UMAP clustering of all 9,360 monocytes revealed 9 distinct monocyte subsets (MS) (Figure 3A). These clusters could be categorized into the three major monocyte subtypes typically identified by flow cytometry: non-classical, intermediate, and classical based on expression of CD14, MAMU-DRA, and FCGR3(CD16) (Supplementary Figure 4A). As shown by flow cytometry, the frequency of these three major subsets was comparable between controls and CHD animals by scRNA-Seq analysis (Supplementary Figure 4B). The non-classical monocytes formed one cluster (MS6) exhibiting high expression of CX3CR1, MS4A7, and FCGR3 and lower expression of CD14 and MHC II molecule $M A M U-D R A$ (Figure 3A, B, and Supplementary Figure 4C). The intermediate monocytes also fell into one cluster (MS4), expressing lower levels of non-classical (MS4A7, FCGR3A) and classical (CD14, S100A8) markers with higher expression of $M A M U-D R A$ (Figures 3A, B, and Supplementary Figure 4C). Finally, 7 clusters of classical monocytes were identified (MS1, MS2, MS3, MS5, MS7, MS8, and MS9), based on expression of CST3, GOS2, S100A8, S100A9, HIF1A, SOD2, EGR1, and HERC5 (Figures 3A, B, and Supplementary Figure 4C). These data reveal considerable, previously unappreciated transcriptional heterogeneity within the classical monocytes. Subsets MS5, MS7 were primarily detected in monocytes from CHD animals while MS8 was primarily detected in monocytes from control animals (Figure 3 and Supplementary Figure 4D). Module scoring revealed that MS5 had the lowest expression of genes that play a role in antigen presentation as well as hypoxia, while MS5 and MS7 had higher expression of genes within the TLRand IL-6- signaling pathways (Supplementary Figure 4E).

To identify the biological implications of these subsets, we extracted the genes that define each cluster and performed functional enrichment (Figure 3C). Although genes that define MS5 (classical - CHD) and MS8 (classical - control) subsets enriched to similar GO terms ("Regulated exocytosis", "granulocyte activation/migration"), only genes highly expressed in MS5 (CHD animals) enriched to "Response to alcohol" and "Wound healing", notably FOSB, HIF1A, and FN1. Interestingly, genes that define MS7 subset (classical $\mathrm{CHD}$ ) enriched to "Cytokine-mediated signaling pathway" including CSF3R, IRF1, IRF7, NFKBIA, STAT1, STAT3, and VEGFA. Classical subsets MS1 and MS2 were slightly more abundant in monocytes from control animals and expressed high levels of CST3 and GOS2 as well as S100A8 and S100A9, respectively.

To better understand the relationships between the classical monocyte clusters and their differentiation/activation states, we performed a trajectory analysis. The monocyte clusters were ordered by pseudotime, starting with the most abundant MS1 cluster which resulted in four unique trajectory lineages (Figure 3D). Lineages 1, 2 and 4 indicate transitions culminating in MS8, MS3, and MS5, respectively. Lineage 3 is the classical to non-classical differentiation trajectory. Density plots revealed enrichment of control monocytes at the start of each lineage (less differentiated) and enrichment of the CHD monocytes at the end of the lineages (more differentiated), most notably in Lineage 4 (Figure 3E).

We next assessed differential gene expression with CHD in the 3 major subsets (Supplementary Figure 4F). This analysis showed that IFN-inducible genes WARS and IDO were upregulated in the classical CHD monocytes, whereas MHC-II gene $M A M U-D R B 1$ was downregulated (Supplementary Figure 4F). In the intermediate monocytes, CHD induced upregulation of inflammatory signaling genes MAP3K and S100P but led to decreased expression of FC-receptor gene FCGR3 (Supplementary Figure 4F). The 33 DEG downregulated in the non-classical monocytes from the CHD group relative to controls enriched to antigen processing and IFN $\gamma$ signaling pathways (Supplementary Figure 4G). Finally, we detected a significant reduction in lysosome and Fc $\gamma$-receptor mediated phagocytosis modules in the CHD monocytes (Figure 3F) while gene modules for $\mathrm{NFkB}$ signaling and HIF1A signaling were increased (Figure 3G).

\section{Chronic Heavy Drinking Impacts the Epigenome of Circulating Monocytes}

To uncover epigenetic basis for the altered transcriptional profile in resting monocytes and altered functional responses with CHD, we profiled the chromatin accessibility in purified resting monocytes from female macaques ( $n=3$ /group) using the assay for transposase-accessible chromatin sequencing (ATAC-Seq) (55). We identified 11,717 differentially accessible regions (DAR) that were open in the CHD monocytes compared to 9,173 DAR that were accessible in the controls (Figure 4A). More than 25\% of the accessible regions in the CHD monocytes mapped to promoter regions and were closer to the transcription start site compared to accessible regions in monocytes from control animals (Figure $\mathbf{4 A}$ and Supplementary Figure 5A). Genes regulated by promoter regions open in the $\mathrm{CHD}$ monocytes enriched to GO terms associated with cytokine production and myeloid cell activation (Figure 4B). Additional analysis using GREAT showed that cis-regulatory elements in the distal intergenic regions open in $\mathrm{CHD}$ monocytes enriched to processes involved with apoptotic signaling, MAPK signaling cascade, and myeloid cell differentiation (Supplementary Figure 5B). Motif enrichment analysis of open chromatin 


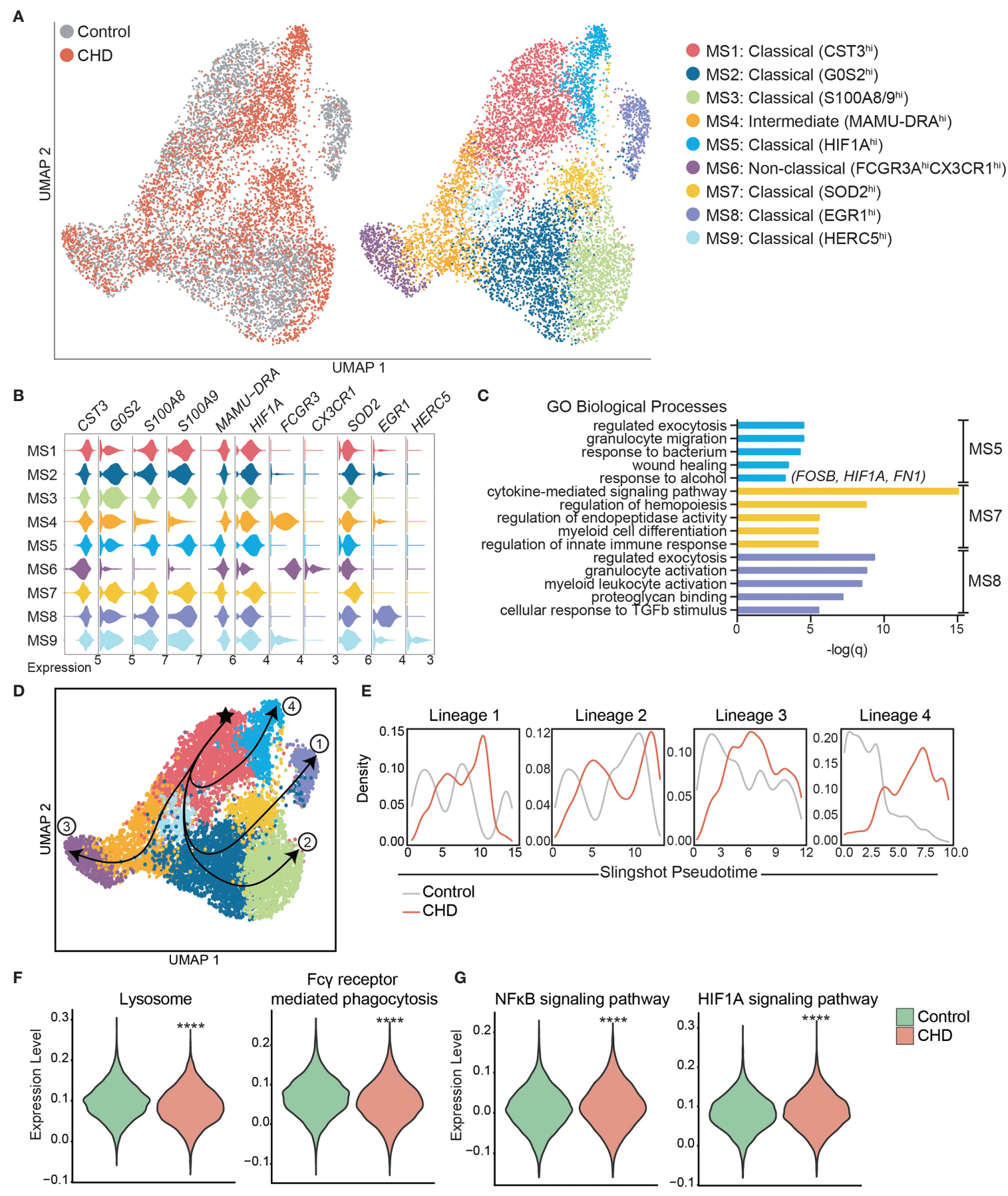

FIGURE 3 | Single cell RNA-Seq analysis of monocytes with CHD. Monocytes ( $n=3$ control/3 CHD) were purified from total PBMC from female macaques and subjected to 10X scRNA-Seq analysis. (A) Visualization of 9,360 monocytes by uniform manifold approximation and projection (UMAP) colored by group (control and CHD) as well as by identified clusters. (B) Stacked violin plots representing expression of key genes used for cluster identification, grouped by monocyte subset cluster. (C) GO Biological Process enrichment from Metascape of genes highly expressed in MS5, MS7, and MS8, defined by q-value. (D) Trajectory analysis of monocytes determined using Slingshot. (E) Cell density plots for Control and CHD groups across each of four trajectory lineages determined by Slingshot. (F, G) Violin plots comparing (F) Lysosome and Fcy receptor-mediated phagocytosis and (G) NFkB signaling and HIF1A signaling pathway module scores in all monocytes of both groups. Statistical analysis of module scores was performed using a t-test with Welch's correction where ${ }^{\star \star \star \star} p<0.0001$. 
A

A Feature Distribution

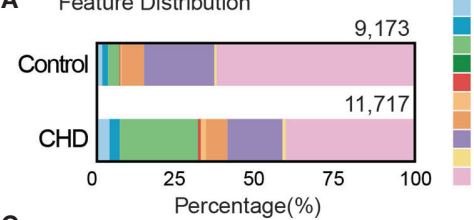

C

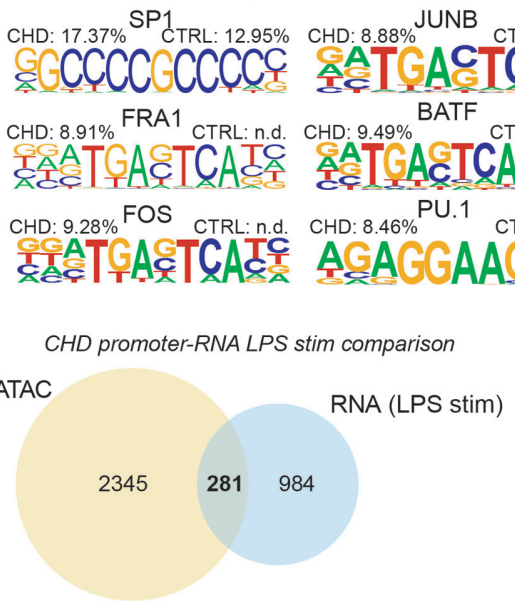

Promoter (1-2kb) B

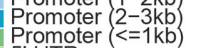

Promoter $(<=1 \mathrm{~kb})$

3' UTR

Other Exon

st Intron

Other Intron

ownstream (<=300)

Distal Intergenic

cran af

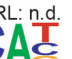

CTRL: n.d.

CTRL: n.d.
B

Response to peptide Neg. regulation of cellular component organization Protein domain specific binding Regulation of cytokine production Positive regulation of organelle organization Regulation of cellular response to stress Ubiquitin-like protein ligase binding Regulation of mitochondrion organization Kinase activity
Negative regulation of cell differentiation Endocytic recycling Response to wounding Myeloid cell activation Response to decreased oxygen levels

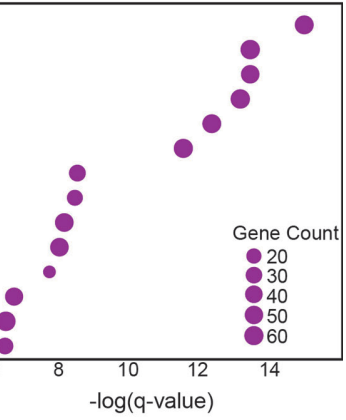

F ATAC-Seq Normalized Reads

E
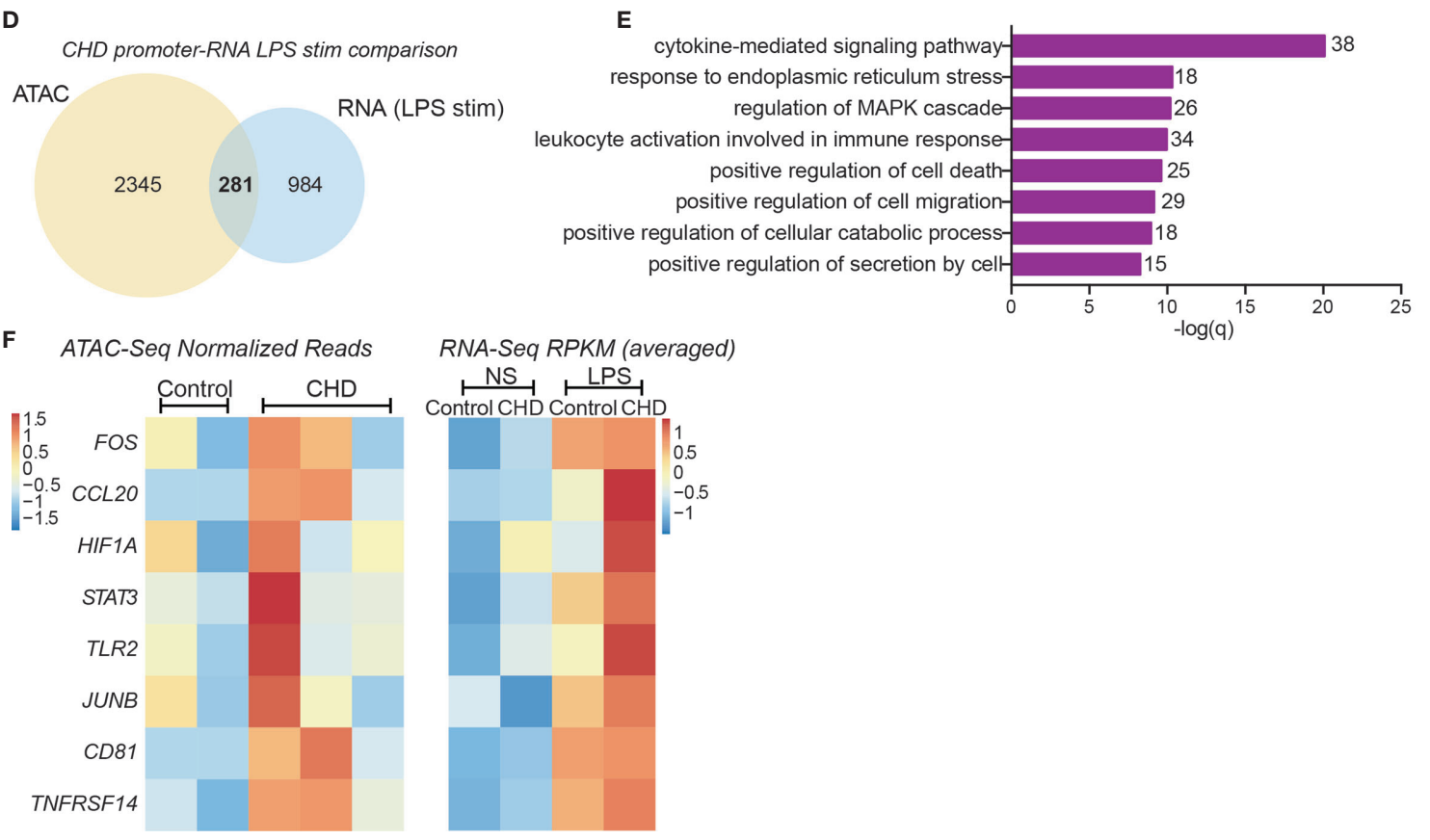

FIGURE 4 | CHD primes the monocyte epigenome for inflammatory response. Monocytes ( $n=2$ Control/3 CHD) were purified from PBMC from female macaques and subjected to ATAC-Seq analysis. (A) Bar plot showing genomic feature distribution of the open chromatin regions (fold-change $\geq 2$ ) in control and $\mathrm{CHD}$ monocytes at resting state. (B) GO Biological process enrichment from Metascape of genes regulated by the open promoter regions ( $\leq 1 \mathrm{~kb}$, fold-change $\geq 3$ ) in $\mathrm{CHD}$ monocytes. The X-axis represents -log(q-value) and the size of the dot represents the number of genes within that term. (C) Homer motif enrichment of the open chromatin regions. All listed motifs have significantly enriched binding sites in the CHD monocytes where the percentage value listed is the percentage of target sequences with that motif. (D) Venn diagram of genes regulated by the open promoter regions ( $\leq 1 \mathrm{~kb}$, fold-change $\geq 3$ ) and DEG detected following LPS stimulation in the CHD monocytes. (E) GO Biological process enrichment terms from Metascape of the 281 overlapping genes from (D). (F) Heatmaps of the normalized expression of open promoter region counts and RPKM from bulk RNA-Seq analysis for selected common genes.

regions in the $\mathrm{CHD}$ and control monocytes demonstrated increased putative binding sites for transcription factors important for monocyte differentiation and activation SP1, FRA1, FOS, JUNB, BATF, and PU.1 with CHD (59-63) (Figure 4C). To link the resting epigenome of the $\mathrm{CHD}$ monocytes to the enhanced transcriptional response after LPS stimulation, we compared the genes associated with the open promoter regions $(<1 \mathrm{~kb})$ and the DEG identified after LPS stimulation and identified 281 common genes (Figure 4D). Functional enrichment of these genes showed significant mapping to Biological Process "Cytokine-mediated signaling" including transcription factors FOS, HIF1A, STAT3 and JUNB (Figure 4E). These genes as well as inflammatory CCL20, TLR2, CD81 and TNFSF14 had both open promoters in the resting $\mathrm{CHD}$ monocytes as well as upregulated gene expression in the LPS-stimulated CHD monocytes, linking open chromatin under resting conditions with CHD to transcriptional LPS responses (Figure $\mathbf{4 F}$ ).

\section{CHD Impairs Monocyte Response to E. coli}

We next asked whether CHD would affect the monocyte response to pathogens. Monocytes from male and female macaques $(n=3$ 
controls; $\mathrm{n}=6 \mathrm{CHD}$ ) were co-cultured with heat-killed E. coli for 16 hours and immune mediator production was measured using Luminex (Supplementary Figure 1A). In contrast to the response to purified LPS, monocytes from CHD animals generated a dampened response to $E$. coli characterized by reduced levels of IL-1 $\beta$ IL-6, MIP-1 $\beta$, and IL-15 and to a lesser extent I-TAC and IL-5 compared to their control counterparts (Figures 5A, B). No sex differences were noted in these assays, indicating that defects in monocyte responses to bacterial pathogens are comparable in male and female macaques. Moreover, these data suggests that CHD differentially impacts pathogen recognition and activation pathways within monocytes.

\section{DISCUSSION}

It is well-appreciated that chronic alcohol drinking exerts a profound impact on peripheral innate immune cells. However, the limitations provided by the complexity of studying alcohol consumption in humans has left major gaps in understanding mechanisms that underlie the immune response under conditions of heavy alcohol drinking. In this study, we utilized a macaque model of voluntary ethanol self-administration to profile peripheral monocytes in animals after 12 months of daily alcohol drinking. We first demonstrated that circulating monocytes from CHD animals generate a hyper-inflammatory response to ex vivo LPS stimulation at the transcript and protein level. A comprehensive profiling of the monocytes by scRNA-Seq and ATAC-Seq revealed alterations in differentiation state as well as epigenetic landscape with CHD. In contrast to what we observed following ex vivo stimulation with LPS, monocytes from CHD animals generated a dampened production of immune mediators following ex vivo E. coli stimulation. This highlights critical aspects of the complexity of immune cell responses and the necessity of utilizing both purified stimulants as well as real pathogens to uncover the underlying mechanisms of response.

The enhanced inflammatory responses in response to ex vivo stimulation with LPS by PBMC from CHD female macaques are in line with our previous data for PBMC from CHD male macaques (41), indicating that this consequence of CHD is sex independent. Our follow up phenotyping revealed that there were in fact more CD14+ monocytes in the PBMC of CHD animals, but no changes were observed in the distribution of classical, non-classical, and intermediate monocyte populations. Further analysis of purified monocytes indicated that this hyperinflammatory response is mediated largely by intrinsic changes within monocytes and not just increased cell numbers. Indeed, CHD induced significant transcriptional differences consistent with activation of inflammatory pathways in monocytes at resting state. Moreover, LPS stimulation resulted in a drastic increase in numbers of DEG from the CHD monocytes with increased expression of genes associated with inflammatory response. In contrast, genes involved in carbohydrate catabolic processes were not upregulated in monocytes from CHD animals suggesting metabolic rewiring associated with a heightened inflammatory state (64). The increased non-specific inflammatory
A

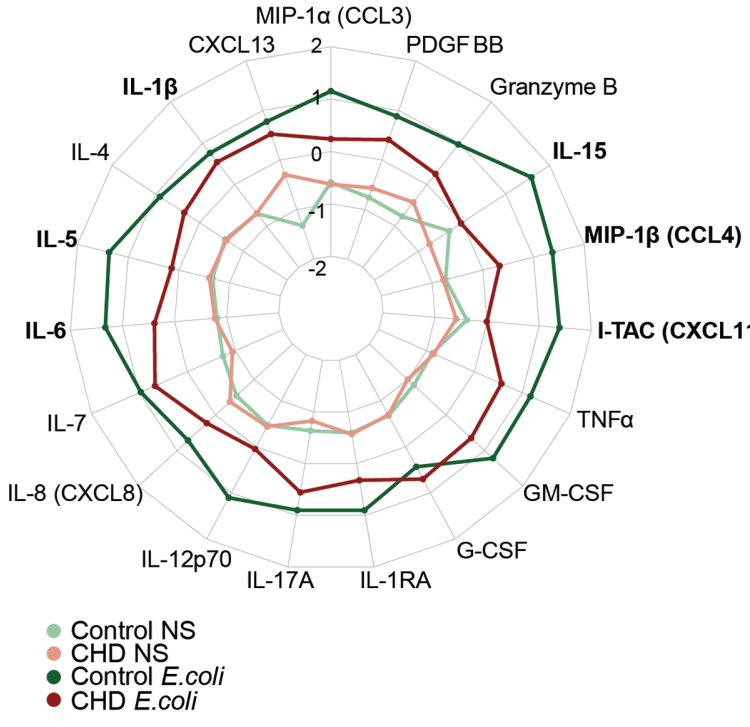

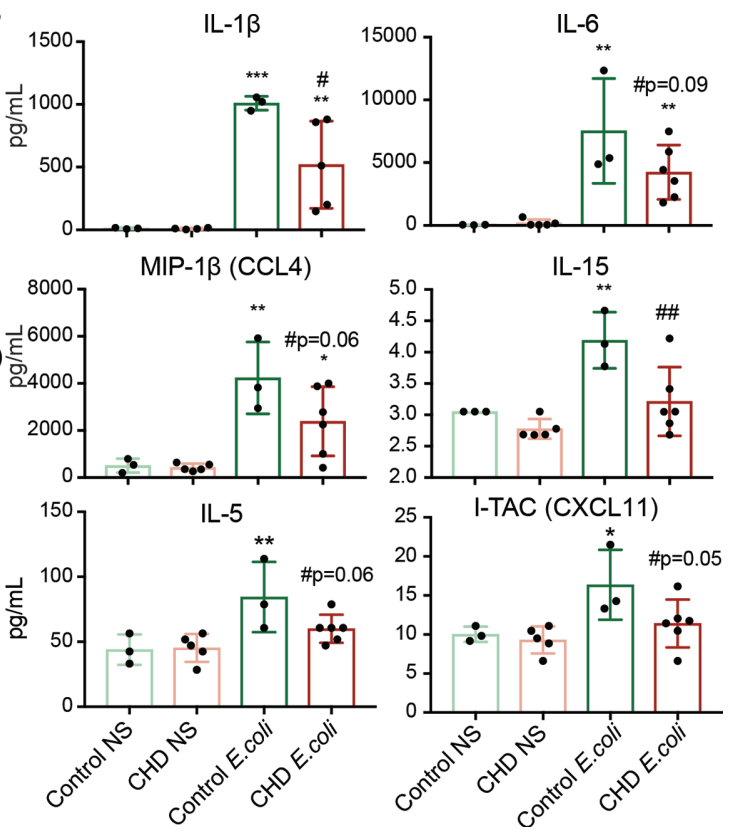

FIGURE 5 | Monocyte response to $E$. coli is dampened with CHD. Purified monocytes ( $n=2$ Control Male, 1 Control Female/2 CHD Male, 4 CHD Female) were stimulated with heat-killed E. coli bacteria for 16 hours. (A) Spider plot representing average Z-scores for each group across the indicated analytes measured by Luminex. (B) Bar plots showing concentration (pg/ml) of selected immune mediators (TNF $\alpha$, IL-6, MIP-1b, IL-15, IL-5, I-TAC). ${ }^{*} \mathrm{p}<0.05,{ }^{* \star} \mathrm{p}<0.01,{ }^{* \star *} \mathrm{p}<0.001$ between $E$. coli and unstimulated condition. If indicated, ${ }^{*}$ is significance between $\mathrm{CHD}$ and control for each stimulation condition. 
mediator production in response to ex vivo LPS stimulation are in line with previous in vitro studies that have reported hyperactivation of myeloid cells with prolonged alcohol exposure. This also fits with hyper-inflammatory phenotypes associated with patients with alcohol use disorders, especially those with alcoholic liver disease $(16,27,65)$.

To elucidate the molecular basis for this dysregulated inflammatory response, we profiled monocytes from CHD and control animals by scRNA-Seq. This analysis revealed tremendous diversity amongst classical monocytes that has not been previously appreciated. We identified two unique classical clusters within monocytes from $\mathrm{CHD}$ animals expressing high levels of hypoxia factor HIF1A (MS5), and antioxidant defense molecule SOD2 (MS7). Alcohol and the products of its metabolism can induce oxidative stress which can alter cellular transcriptional profiles, potentially explaining the profile of the MS5 cluster (66). CHD animals also had a higher number of cells within the anti-viral cluster MS9 that expressed high levels of interferon stimulated (ISG) genes such as HERC5. This observation is in line with our earlier study that reported higher levels of ISG within PBMC from these same animals (18). Functional module scoring of all the monocytes revealed downregulation of genes involved in lysosome function and Fc $\gamma$ receptor mediated phagocytosis, but upregulation of $\mathrm{NF \kappa B}$ signaling and HIF1A signaling. Moreover, trajectory within the monocyte subsets revealed that CHD accelerates differentiation/ activation of classical monocyte subsets making them potentially poised towards a hyper-inflammatory response.

Although the non-classical and intermediate monocytes fell into single clusters with equal numbers of cells from controls and CHD animals, differential gene expression analysis revealed significant downregulation of genes associated with MHC class II, antigen processing and presentation, and IFN $\gamma$ signaling pathways in the CHD monocytes. This observation provides a potential explanation for the reduced response to vaccination observed in CHD animals (67). Further, monocytes from CHD male and female macaques generated a dampened cytokine and chemokine response to heat killed E. coli. This was an unexpected outcome since stimulation with LPS resulted in a heightened inflammatory response and suggests a rewiring of monocytes with CHD towards non-specific inflammatory responses and away from anti-microbial functions. This discrepancy could be explained by the fact that LPS signals exclusively through TLR4, whereas heat killed $E$. coli could trigger a number of TLRs including TLR2/6 (68). Additionally, CHD interferes with phagocytosis and ROS production in macrophages (69), which could interfere with pathogen sensing. While the mechanisms underlying the differences between the heightened response to LPS and dampened response to whole organisms is complex and require further study, our observations provide potential explanation for the reported increased susceptibility of patients with alcohol use disorders to bacterial infections $(10,11)$.

Ethanol metabolites notably acetaldehyde and acetate in addition to minor byproducts such as reactive oxygen species (ROS) and lipid metabolism products can modulate gene expression by binding transcription factors and/or modifying chromatin accessibility (66). Therefore, we profiled chromatin accessibility of monocytes from control and CHD animals by ATAC-Seq. We discovered that promoter regions that regulate genes important for cytokine production and myeloid cell activation were more accessible in monocytes from $\mathrm{CHD}$ animals. These open regions contained putative binding sites for transcription factors important for monocyte activation and differentiation. Integrative analyses showed correlation between chromatin accessibility and expression levels of key inflammatory genes in CHD monocytes, including FOS, HIF1A, and JUNB. This suggests that the monocytes are poised epigenetically towards heightened inflammatory responses.

One proposed mechanism of this hyper-inflammatory state in monocytes $(33,35-37)$ and macrophages with CHD is the translocation of bacterial products into circulation through impaired gut barrier caused by ethanol consumption (34). We detected modestly elevated levels of LAL in circulation but no changes in IgM bound endotoxin. A small increase in these circulating bacterial products could significantly impact the activation state of monocytes; however, it is difficult to tease these effects out from circulating ethanol and its metabolic products (acetaldehyde, acetate). Additionally, liver enzyme levels remained within normal ranges for the duration of the study. Long term exposure to activating agents, like LPS, is believed to lead to tolerance and a decreased response to secondary stimulation in monocytes and macrophages defined by specific changes in chromatin structure (70-73). Interestingly, we see enhanced inflammatory responses of monocytes and macrophages from CHD animals with LPS, akin to innate immune training $(74,75)$. Indeed, ethanol and its metabolites have been found to directly act on histone modifications, creating changes in accessible chromatin $(66,76,77)$. This could be occurring in circulating monocytes, tissue macrophages, or the progenitors of these cells in the bone marrow; perhaps on all three levels, altering the epigenetic structure and therefore functional capacities of these cells (78). In fact, observations in patients with alcohol use disorders suggest an effect of alcohol on hematopoiesis (79). Because blood monocytes are under constant repopulation from bone marrow progenitors, it is conceivable that the progenitors themselves or the process of myelopoiesis are disrupted with CHD.

In summary, this study provides a novel, in-depth, and integrative analysis of the effects of long-term in vivo alcohol drinking on monocytes in non-human primates. Our findings indicate that alcohol drinking alters the baseline epigenetic and transcriptional states of monocytes, which is associated with heightened inflammatory responses but reduced anti-microbial activity. Future studies will focus on linking epigenetic modifications to functional outcomes and investigating the impact of alcohol drinking on bone marrow progenitors.

\section{DATA AVAILABILITY STATEMENT}

The datasets presented in this study can be found in online repositories. The names of the repository/repositories and 
accession number(s) can be found below: (https://www.ncbi.nlm. nih.gov/, PRJNA723053).

\section{ETHICS STATEMENT}

The study was approved by the Institutional Animal Care and Use Committee at the Oregon National Primate Research Center.

\section{AUTHOR CONTRIBUTIONS}

SL, KG, and IM conceived and designed the experiments. SL, SS, and $\mathrm{BD}$ performed the experiments. SL and BD analyzed the data. SL and IM wrote the paper. All authors contributed to the article and approved the submitted version.

\section{FUNDING}

This study was supported by NIH 1R01AA028735-01 (Messaoudi), 5U01AA013510-20 (Grant), and 2R24AA01943111 (Grant). SL is supported by NIH 1F31A028704-01. The content is solely the responsibility of the authors and does not necessarily represent the official views of the NIH.

\section{ACKNOWLEDGMENTS}

We are grateful to the members of the Grant laboratory for expert animal care and sample procurement. We thank Dr. Jennifer Atwood for assistance with sorting in the flow cytometry core at the Institute for Immunology, UCI. We thank Dr. Melanie Oakes from UCI Genomics and HighThroughput Facility for assistance with 10X library preparation and sequencing.

\section{SUPPLEMENTARY MATERIAL}

The Supplementary Material for this article can be found online at: https://www.frontiersin.org/articles/10.3389/fimmu.2021. 724015/full\#supplementary-material

\section{REFERENCES}

1. O'Keefe JH, Bhatti SK, Bajwa A, DiNicolantonio JJ, Lavie CJ. Alcohol and Cardiovascular Health: The Dose Makes the Poison...or the Remedy. Mayo Clin Proc (2014) 89:382-93. doi: 10.1016/j.mayocp.2013.11.005

2. Mukamal KJ, Rimm EB. Alcohol's Effects on the Risk for Coronary Heart Disease. Alcohol Res Health (2001) 25:255-61.

3. Fedirko V, Tramacere I, Bagnardi V, Rota M, Scotti L, Islami F, et al. Alcohol Drinking and Colorectal Cancer Risk: An Overall and Dose-Response MetaAnalysis of Published Studies. Ann Oncol (2011) 22:1958-72. doi: 10.1093/ annonc/mdq653
Supplementary Figure 1 I (A) Experimental Design figure for this study. (B) Scatter plots showing Spearman correlation between average EtOH dose (grams EtOH/kg body weight/day) and concentration $(\mathrm{pg} / \mathrm{ml}$ ) of the secreted factors IL-6, IL15, IL-23, PD-L1. (C) Heatmap of genes related to Regulation of transcription factor activity and Lymphocyte activation upregulated in CHD PBMC with LPS. (D) Heatmap of genes involved in Chemokine production and Carbohydrate catabolic process pathways downregulated in CHD PBMC with LPS.

Supplementary Figure 2 | (A) Gating strategy used to identify the three populations of monocytes in the blood. (B) Relative percentages of the three monocyte populations in the controls and CHD macaques. (C) $\log _{2}$ median fluorescence intensities (MFI) of monocyte cell surface markers across the controls and CHD macaques. (D) Heatmap of normalized expression of differentially expressed genes between resting control and CHD monocytes. (E, F) LAL (E) and IgM-bound endotoxin (F) levels measured from plasma by ELISA. (G) Alanine transaminase (ALT) and aspartate transaminase (AST) were measured to test for overt liver damage. T-test with Welch's correction was used to measure significance.

Supplementary Figure 3 | (A) Bubble plot representing immune factor production $(\mathrm{pg} / \mathrm{ml})$ in the presence or absence of LPS stimulation of monocytes from control and $\mathrm{CHD}$ animals. The size of each circle represents the indicates the $\log _{2}$ mean concentration of the indicated secreted factor and the color denotes the $-\log _{2}$ transformed $p$ value with the darkest pink representing the most significant value. The $p$-values were calculated between the unstimulated and stimulated conditions for each group using One-way ANOVA and a p-value cut-off of 0.05 was set. White circles indicate non-significant p-value. (B) Principal component analysis (PCA) of $\mathrm{CHD}$ and control monocytes under resting, unstimulated (6 hours of media) and LPS stimulation (6 hours) conditions. (C, D) Volcano plot representing up- and downregulated DEG with CHD in non-stimulated (C) and LPS stimulated (D) monocytes. Red = significant with an FDR $\leq 0.05$ and fold change $\geq 2$. (E) Bubble plot representing transcription factors predicted to regulate LPS-responsive DEGs. The size of the dot represents the number of genes and the color represents Chea3 score where lower number is more significant. Analysis was performed using the Chea3 web browser.

Supplementary Figure 4 | (A) Feature plots showing relative gene expression of canonical monocyte markers CD14, MAMU-DRA, and FCGR3 in all monocytes. (B) Stacked bar graph depicting relative abundance of non-classical, intermediate, and classical monocytes in control and CHD monocytes. (C) Heatmap showing relative gene expression of the representative genes used for clustering and subset identification. (D) Stacked bar graph depicting abundance of cells clustered in each monocyte subset in control and CHD groups. (E) Violin plots comparing antigen presentation, IL-6 signaling, hypoxia, and TLR signaling module scores with the classical monocyte subsets. (F) Volcano plots of the up-and downregulated genes comparing $\mathrm{CHD}$ to control non-classical, intermediate, and classical monocytes. The purple color indicates significant DEG where FDR $\leq 0.05$ and fold-change $\geq 2$. (G) Bar graph showing functional enrichment pathways of DEG downregulated with $\mathrm{CHD}$ in the non-classical monocyte cluster (MS6), defined by q-value.

Supplementary Figure 5 | (A) Distribution of the open chromatin regions in the $\mathrm{CHD}$ and control monocytes relative to the transcription start site (TSS). (B) Top GO Biological process enrichment of the genes predicted to be cis-regulated by nonpromoter regions more open with $\mathrm{CHD}$. Genomic regions were lifted over from rhesus macaque to homo sapien genome (UCSC) and further enriched using the GREAT.

4. Baan R, Straif K, Grosse Y, Secretan B, El Ghissassi F, Bouvard V, et al. Carcinogenicity of Alcoholic Beverages. Lancet Oncol (2007) 8:292-3. doi: 10.1016/S1470-2045(07)70099-2

5. Grewal P, Viswanathen VA. Liver Cancer and Alcohol. Clin Liver Dis (2012) 16:839-50. doi: 10.1016/j.cld.2012.08.011

6. Priddy BM, Carmack SA, Thomas LC, Vendruscolo JC, Koob GF, Vendruscolo LF. Sex, Strain, and Estrous Cycle Influences on Alcohol Drinking in Rats. Pharmacol Biochem Behav (2017) 152:61-7. doi: 10.1016/ j.pbb.2016.08.001

7. Bruha R, Dvorak K, Petrtyl J. Alcoholic Liver Disease. World J Hepatol (2012) 4:81-90. doi: 10.4254/wjh.v4.i3.81 
8. O’Brien JMJr., Lu B, Ali NA, Levine DA, Aberegg SK, Lemeshow S. Insurance Type and Sepsis-Associated Hospitalizations and Sepsis-Associated Mortality Among US Adults: A Retrospective Cohort Study. Crit Care (2011) 15:R130. doi: $10.1186 / \mathrm{cc} 10243$

9. Mokdad AH, Marks JS, Stroup DF, Gerberding JL. Actual Causes of Death in the United States, 2000. JAMA (2004) 291:1238-45. doi: 10.1001/jama.291.10.1238

10. Delgado-Rodriguez M, Mariscal-Ortiz M, Gomez-Ortega A, MartinezGallego G, Palma-Perez S, Sillero-Arenas M, et al. Alcohol Consumption and the Risk of Nosocomial Infection in General Surgery. Br J Surg (2003) 90:1287-93. doi: 10.1002/bjs.4186

11. Saitz R, Ghali WA, Moskowitz MA. The Impact of Alcohol-Related Diagnoses on Pneumonia Outcomes. Arch Intern Med (1997) 157:1446-52. doi: 10.1001/ archinte.157.13.1446

12. Baum MK, Rafie C, Lai S, Sales S, Page JB, Campa A. Alcohol Use Accelerates HIV Disease Progression. AIDS Res Hum Retroviruses (2010) 26:511-8. doi: 10.1089/aid.2009.0211

13. Bhattacharya R, Shuhart MC. Hepatitis C and Alcohol: Interactions, Outcomes, and Implications. J Clin Gastroenterol (2003) 36:242-52. doi: 10.1097/00004836-200303000-00012

14. Delgado-Rodriguez M, Gomez-Ortega A, Mariscal-Ortiz M, Palma-Perez S, Sillero-Arenas M. Alcohol Drinking as a Predictor of Intensive Care and Hospital Mortality in General Surgery: A Prospective Study. Addiction (2003) 98:611-6. doi: 10.1046/j.1360-0443.2003.00353.x

15. Jung MK, Callaci JJ, Lauing KL, Otis JS, Radek KA, Jones MK, et al. Alcohol Exposure and Mechanisms of Tissue Injury and Repair. Alcohol Clin Exp Res (2011) 35:392-9. doi: 10.1111/j.1530-0277.2010.01356.x

16. Szabo G, Saha B. Alcohol's Effect on Host Defense. Alcohol Res (2015) 37:159-70.

17. Sureshchandra S, Stull C, Ligh BJK, Nguyen SB, Grant KA, Messaoudi I. Chronic Heavy Drinking Drives Distinct Transcriptional and Epigenetic Changes in Splenic Macrophages. EBioMedicine (2019) 43:594-606. doi: 10.1016/j.ebiom.2019.04.027

18. Sureshchandra S, Rais M, Stull C, Grant K, Messaoudi I. Transcriptome Profiling Reveals Disruption of Innate Immunity in Chronic Heavy Ethanol Consuming Female Rhesus Macaques. PloS One (2016) 11:e0159295. doi: 10.1371/journal.pone.0159295

19. Teh YC, Ding JL, Ng LG, Chong SZ. Capturing the Fantastic Voyage of Monocytes Through Time and Space. Front Immunol (2019) 10:834. doi: 10.3389/fimmu.2019.00834

20. Guilliams M, Mildner A, Yona S. Developmental and Functional Heterogeneity of Monocytes. Immunity (2018) 49:595-613. doi: 10.1016/ j.immuni.2018.10.005

21. Norkina O, Dolganiuc A, Shapiro T, Kodys K, Mandrekar P, Szabo G. Acute Alcohol Activates STAT3, AP-1, and Sp-1 Transcription Factors via the Family of Src Kinases to Promote IL-10 Production in Human Monocytes. J Leukoc Biol (2007) 82:752-62. doi: 10.1189/jlb.0207099

22. Pang M, Bala S, Kodys K, Catalano D, Szabo G. Inhibition of TLR8- and TLR4-Induced Type I IFN Induction by Alcohol is Different From its Effects on Inflammatory Cytokine Production in Monocytes. BMC Immunol (2011) 12:55. doi: 10.1186/1471-2172-12-55

23. Bala S, Tang A, Catalano D, Petrasek J, Taha O, Kodys K, et al. Induction of Bcl-3 by Acute Binge Alcohol Results in Toll-Like Receptor 4/LPS Tolerance. J Leukoc Biol (2012) 92:611-20. doi: 10.1189/jlb.0112050

24. Pruett SB, Fan R, Zheng Q, Schwab C. Differences in IL-10 and IL-12 Production Patterns and Differences in the Effects of Acute Ethanol Treatment on Macrophages In Vivo and In Vitro. Alcohol (2005) 37:1-8. doi: 10.1016/j.alcohol.2005.09.004

25. Afshar M, Richards S, Mann D, Cross A, Smith GB, Netzer G, et al. Acute Immunomodulatory Effects of Binge Alcohol Ingestion. Alcohol (2015) 49:5764. doi: 10.1016/j.alcohol.2014.10.002

26. Zhang Z, Bagby GJ, Stoltz D, Oliver P, Schwarzenberger PO, Kolls JK. Prolonged Ethanol Treatment Enhances Lipopolysaccharide/Phorbol Myristate Acetate-Induced Tumor Necrosis Factor-Alpha Production in Human Monocytic Cells. Alcohol Clin Exp Res (2001) 25:444-9. doi: 10.1111/j.1530-0277.2001.tb02233.x

27. Mandrekar P, Bala S, Catalano D, Kodys K, Szabo G. The Opposite Effects of Acute and Chronic Alcohol on Lipopolysaccharide-Induced Inflammation are Linked to IRAK-M in Human Monocytes. J Immunol (2009) 183:1320-7. doi: 10.4049/jimmunol.0803206
28. Maraslioglu M, Oppermann E, Blattner C, Weber R, Henrich D, Jobin C, et al. Chronic Ethanol Feeding Modulates Inflammatory Mediators, Activation of Nuclear Factor-Kappab, and Responsiveness to Endotoxin in Murine Kupffer Cells and Circulating Leukocytes. Mediators Inflamm (2014) 2014:808695. doi: 10.1155/2014/808695

29. Coleman LGJr., Crews FT. Innate Immune Signaling and Alcohol Use Disorders. Handb Exp Pharmacol (2018) 248:369-96. doi: 10.1007/ 164_2018_92

30. O'Halloran EB, Curtis BJ, Afshar M, Chen MM, Kovacs EJ, Burnham EL. Alveolar Macrophage Inflammatory Mediator Expression is Elevated in the Setting of Alcohol Use Disorders. Alcohol (2016) 50:43-50. doi: 10.1016/ j.alcohol.2015.11.003

31. Zhu X, Coleman RA, Alber C, Ballas ZK, Waldschmidt TJ, Ray NB, et al. Chronic Ethanol Ingestion by Mice Increases Expression of CD80 and CD86 by Activated Macrophages. Alcohol (2004) 32:91-100. doi: 10.1016/ j.alcohol.2004.01.004

32. McClain CJ, Cohen DA. Increased Tumor Necrosis Factor Production by Monocytes in Alcoholic Hepatitis. Hepatology (1989) 9:349-51. doi: 10.1002/ hep. 1840090302

33. Bird GL, Sheron N, Goka AK, Alexander GJ, Williams RS. Increased Plasma Tumor Necrosis Factor in Severe Alcoholic Hepatitis. Ann Intern Med (1990) 112:917-20. doi: 10.7326/0003-4819-112-12-917

34. Bishehsari F, Magno E, Swanson G, Desai V, Voigt RM, Forsyth CB, et al. Alcohol and Gut-Derived Inflammation. Alcohol Res (2017) 38:163-71.

35. Mukherjee S. Alcoholism and its Effects on the Central Nervous System. Curr Neurovasc Res (2013) 10:256-62. doi: 10.2174/15672026113109990004

36. Szabo G, Lippai D. Converging Actions of Alcohol on Liver and Brain Immune Signaling. Int Rev Neurobiol (2014) 118:359-80. doi: 10.1016/ B978-0-12-801284-0.00011-7

37. Mehta AJ, Guidot DM. Alcohol and the Lung. Alcohol Res (2017) 38:243-54.

38. Stampfer MJ, Colditz GA, Willett WC, Manson JE, Arky RA, Hennekens CH, et al. A Prospective Study of Moderate Alcohol Drinking and Risk of Diabetes in Women. Am J Epidemiol (1988) 128:549-58. doi: 10.1093/ oxfordjournals.aje.a115002

39. Baker EJ, Farro J, Gonzales S, Helms C, Grant KA. Chronic Alcohol SelfAdministration in Monkeys Shows Long-Term Quantity/Frequency Categorical Stability. Alcohol Clin Exp Res (2014) 38:2835-43. doi: 10.1111/ acer. 12547

40. Grant KA, Leng X, Green HL, Szeliga KT, Rogers LS, Gonzales SW. Drinking Typography Established by Scheduled Induction Predicts Chronic Heavy Drinking in a Monkey Model of Ethanol Self-Administration. Alcohol Clin Exp Res (2008) 32:1824-38. doi: 10.1111/j.1530-0277.2008.00765.x

41. Sureshchandra S, Raus A, Jankeel A, Ligh BJK, Walter NAR, Newman N, et al. Dose-Dependent Effects of Chronic Alcohol Drinking on Peripheral Immune Responses. Sci Rep (2019) 9:7847. doi: 10.1038/s41598-019-44302-3

42. Jimenez VA, Helms CM, Cornea A, Meshul CK, Grant KA. An Ultrastructural Analysis of the Effects of Ethanol Self-Administration on the Hypothalamic Paraventricular Nucleus in Rhesus Macaques. Front Cell Neurosci (2015) 9:260. doi: $10.3389 /$ fncel.2015.00260

43. Barr T, Sureshchandra S, Ruegger P, Zhang J, Ma W, Borneman J, et al. Concurrent Gut Transcriptome and Microbiota Profiling Following Chronic Ethanol Consumption in Nonhuman Primates. Gut Microbes (2018) 9:33856. doi: 10.1080/19490976.2018.1441663

44. Trapnell C, Pachter L, Salzberg SL. TopHat: Discovering Splice Junctions With RNA-Seq. Bioinformatics (2009) 25:1105-11. doi: 10.1093/ bioinformatics/btp120

45. Langmead B, Salzberg SL. Fast Gapped-Read Alignment With Bowtie 2. Nat Methods (2012) 9:357-9. doi: 10.1038/nmeth.1923

46. Lawrence M, Huber W, Pages H, Aboyoun P, Carlson M, Gentleman R, et al. Software for Computing and Annotating Genomic Ranges. PloS Comput Biol (2013) 9:e1003118. doi: 10.1371/journal.pcbi.1003118

47. Robinson MD, McCarthy DJ, Smyth GK. Edger: A Bioconductor Package for Differential Expression Analysis of Digital Gene Expression Data. Bioinformatics (2010) 26:139-40. doi: 10.1093/bioinformatics/btp616

48. Zhou Y, Zhou B, Pache L, Chang M, Khodabakhshi AH, Tanaseichuk O, et al. Metascape Provides a Biologist-Oriented Resource for the Analysis of Systems-Level Datasets. Nat Commun (2019) 10:1523. doi: 10.1038/s41467019-09234-6 
49. Huang DW, Sherman BT, Tan Q, Collins JR, Alvord WG, Roayaei J, et al. The DAVID Gene Functional Classification Tool: A Novel Biological ModuleCentric Algorithm to Functionally Analyze Large Gene Lists. Genome Biol (2007) 8:R183. doi: 10.1186/gb-2007-8-9-r183

50. Shannon P, Markiel A, Ozier O, Baliga NS, Wang JT, Ramage D, et al. Cytoscape: A Software Environment for Integrated Models of Biomolecular Interaction Networks. Genome Res (2003) 13:2498-504. doi: 10.1101/ gr.1239303

51. Keenan AB, Torre D, Lachmann A, Leong AK, Wojciechowicz ML, Utti V, et al. ChEA3: Transcription Factor Enrichment Analysis by Orthogonal Omics Integration. Nucleic Acids Res (2019) 47:W212-24. doi: 10.1093/nar/ gkz446

52. Zheng GX, Terry JM, Belgrader P, Ryvkin P, Bent ZW, Wilson R, et al. Massively Parallel Digital Transcriptional Profiling of Single Cells. Nat Commun (2017) 8:14049. doi: 10.1038/ncomms14049

53. Butler A, Hoffman P, Smibert P, Papalexi E, Satija R. Integrating Single-Cell Transcriptomic Data Across Different Conditions, Technologies, and Species. Nat Biotechnol (2018) 36:411-20. doi: 10.1038/nbt.4096

54. Street K, Risso D, Fletcher RB, Das D, Ngai J, Yosef N, et al. Slingshot: Cell Lineage and Pseudotime Inference for Single-Cell Transcriptomics. BMC Genomics (2018) 19:477. doi: 10.1186/s12864-018-4772-0

55. Corces MR, Trevino AE, Hamilton EG, Greenside PG, Sinnott-Armstrong NA, Vesuna S, et al. An Improved ATAC-Seq Protocol Reduces Background and Enables Interrogation of Frozen Tissues. Nat Methods (2017) 14:959-62. doi: $10.1038 /$ nmeth.4396

56. Heinz S, Benner C, Spann N, Bertolino E, Lin YC, Laslo P, et al. Simple Combinations of Lineage-Determining Transcription Factors Prime CisRegulatory Elements Required for Macrophage and B Cell Identities. Mol Cell (2010) 38:576-89. doi: 10.1016/j.molcel.2010.05.004

57. Yu G, Wang LG, He QY. ChIPseeker: An R/Bioconductor Package for ChIP Peak Annotation, Comparison and Visualization. Bioinformatics (2015) 31:2382-3. doi: 10.1093/bioinformatics/btv145

58. Liao Y, Smyth GK, Shi W. Featurecounts: An Efficient General Purpose Program for Assigning Sequence Reads to Genomic Features. Bioinformatics (2014) 30:923-30. doi: 10.1093/bioinformatics/btt656

59. Fontana MF, Baccarella A, Pancholi N, Pufall MA, Herbert DR, Kim CC. JUNB is a Key Transcriptional Modulator of Macrophage Activation. J Immunol (2015) 194:177-86. doi: 10.4049/jimmunol.1401595

60. Hannemann N, Cao S, Eriksson D, Schnelzer A, Jordan J, Eberhardt M, et al. Transcription Factor Fra-1 Targets Arginase-1 to Enhance MacrophageMediated Inflammation in Arthritis. J Clin Invest (2019) 129:2669-84. doi: 10.1172/JCI96832

61. Sadeghi K, Wisgrill L, Wessely I, Diesner SC, Schuller S, Durr C, et al. GMCSF Down-Regulates TLR Expression via the Transcription Factor PU.1 in Human Monocytes. PloS One (2016) 11:e0162667. doi: 10.1371/ journal.pone. 0162667

62. Sanchez-Martin L, Estecha A, Samaniego R, Sanchez-Ramon S, Vega MA, Sanchez-Mateos P. The Chemokine CXCL12 Regulates MonocyteMacrophage Differentiation and RUNX3 Expression. Blood (2011) 117:8897. doi: 10.1182/blood-2009-12-258186

63. Zhang DE, Hetherington CJ, Chen HM, Tenen DG. The Macrophage Transcription Factor PU.1 Directs Tissue-Specific Expression of the Macrophage Colony-Stimulating Factor Receptor. Mol Cell Biol (1994) 14:373-81. doi: $10.1128 / \mathrm{mcb} .14 .1 .373-381.1994$

64. Zhu X, Meyers A, Long D, Ingram B, Liu T, Yoza BK, et al. Frontline Science: Monocytes Sequentially Rewire Metabolism and Bioenergetics During an Acute Inflammatory Response. J Leukoc Biol (2019) 105:215-28. doi: 10.1002/ JLB.3HI0918-373R

65. Barr T, Helms C, Grant K, Messaoudi I. Opposing Effects of Alcohol on the Immune System. Prog Neuropsychopharmacol Biol Psychiatry (2016) 65:24251. doi: 10.1016/j.pnpbp.2015.09.001
66. Zakhari S. Alcohol Metabolism and Epigenetics Changes. Alcohol Res (2013) 35:6-16.

67. Messaoudi I, Asquith M, Engelmann F, Park B, Brown M, Rau A, et al. Moderate Alcohol Consumption Enhances Vaccine-Induced Responses in Rhesus Macaques. Vaccine (2013) 32:54-61. doi: 10.1016/j.vaccine.2013.10.076

68. Kawasaki T, Kawai T. Toll-Like Receptor Signaling Pathways. Front Immunol (2014) 5:461. doi: 10.3389/fimmu.2014.00461

69. Karavitis J, Kovacs EJ. AndMacrophage Phagocytosis: Effects of Environmental Pollutants, Alcohol, Cigarette Smoke, and Other External Factors. J Leukoc Biol (2011) 90:1065-78. doi: 10.1189/jlb.0311114

70. Huber R, Bikker R, Welz B, Christmann M, Brand K. TNF Tolerance in Monocytes and Macrophages: Characteristics and Molecular Mechanisms. J Immunol Res (2017) 2017:9570129. doi: 10.1155/2017/9570129

71. Ifrim DC, Quintin J, Joosten LA, Jacobs C, Jansen T, Jacobs L, et al. Trained Immunity or Tolerance: Opposing Functional Programs Induced in Human Monocytes After Engagement of Various Pattern Recognition Receptors. Clin Vaccine Immunol (2014) 21:534-45. doi: 10.1128/CVI.00688-13

72. Seeley JJ, Ghosh S. Molecular Mechanisms of Innate Memory and Tolerance to LPS. J Leukoc Biol (2017) 101:107-19. doi: 10.1189/jlb.3MR0316-118RR

73. Widdrington JD, Gomez-Duran A, Pyle A, Ruchaud-Sparagano MH, Scott J, Baudouin SV, et al. Exposure of Monocytic Cells to Lipopolysaccharide Induces Coordinated Endotoxin Tolerance, Mitochondrial Biogenesis, Mitophagy, and Antioxidant Defenses. Front Immunol (2018) 9:2217. doi: 10.3389/fimmu.2018.02217

74. Saeed S, Quintin J, Kerstens HH, Rao NA, Aghajanirefah A, Matarese F, et al. AndEpigenetic Programming of Monocyte-to-Macrophage Differentiation and Trained Innate Immunity. Science (2014) 345:1251086. doi: 10.1126/ science. 1251086

75. Netea MG, Quintin J, van der Meer JW. Trained Immunity: A Memory for Innate Host Defense. Cell Host Microbe (2011) 9:355-61. doi: 10.1016/ j.chom.2011.04.006

76. de Diego I, Muller-Eigner A, Peleg S. The Brain Epigenome Goes Drunk: Alcohol Consumption Alters Histone Acetylation and Transcriptome. Trends Biochem Sci (2020) 45:93-5. doi: 10.1016/j.tibs.2019.11.002

77. Mews P, Egervari G, Nativio R, Sidoli S, Donahue G, Lombroso SI, et al. Alcohol Metabolism Contributes to Brain Histone Acetylation. Nature (2019) 574:717-21. doi: 10.1038/s41586-019-1700-7

78. Schultze JL, Mass E, Schlitzer A. Emerging Principles in Myelopoiesis at Homeostasis and During Infection and Inflammation. Immunity (2019) 50:288-301. doi: 10.1016/j.immuni.2019.01.019

79. Di Rocco G, Baldari S, Pani G, Toietta G. Stem Cells Under the Influence of Alcohol: Effects of Ethanol Consumption on Stem/Progenitor Cells. Cell Mol Life Sci (2019) 76:231-44. doi: 10.1007/s00018-018-2931-8

Conflict of Interest: The authors declare that the research was conducted in the absence of any commercial or financial relationships that could be construed as a potential conflict of interest.

Publisher's Note: All claims expressed in this article are solely those of the authors and do not necessarily represent those of their affiliated organizations, or those of the publisher, the editors and the reviewers. Any product that may be evaluated in this article, or claim that may be made by its manufacturer, is not guaranteed or endorsed by the publisher.

Copyright (1) 2021 Lewis, Sureshchandra, Doratt, Jimenez, Stull, Grant and Messaoudi. This is an open-access article distributed under the terms of the Creative Commons Attribution License (CC BY). The use, distribution or reproduction in other forums is permitted, provided the original author(s) and the copyright owner(s) are credited and that the original publication in this journal is cited, in accordance with accepted academic practice. No use, distribution or reproduction is permitted which does not comply with these terms. 